See discussions, stats, and author profiles for this publication at: https://www.researchgate.net/publication/338438980

\title{
Review Dynamics and Their Impact on Software Quality
}

Article in IEEE Transactions on Software Engineering · January 2020

DOI: 10.1109/TSE.2020.2964660

CITATIONS

194

2 authors:

(1) Patanamon Thongtanunam

University of Melbourne

20 PUBLICATIONS 373 CITATIONS

SEE PROFILE

Some of the authors of this publication are also working on these related projects:

Project Intelligent Operations of Large-Scale Software Systems View project

Software Ecosystems View project 


\title{
Review Dynamics and Their Impact on Software Quality
}

\author{
Patanamon Thongtanunam, Member, IEEE, and Ahmed E. Hassan, Member, IEEE
}

\begin{abstract}
Code review is a crucial activity for ensuring the quality of software products. Unlike the traditional code review process of the past where reviewers independently examine software artifacts, contemporary code review processes allow teams to collaboratively examine and discuss proposed patches. While the visibility of reviewing activities including review discussions in a contemporary code review tends to increase developer collaboration and openness, little is known whether such visible information influences the evaluation decision of a reviewer or not (i.e., knowing others' feedback about the patch before providing ones own feedback). Therefore, in this work, we set out to investigate the review dynamics, i.e., a practice of providing a vote to accept a proposed patch, in a code review process. To do so, we first characterize the review dynamics by examining the relationship between the evaluation decision of a reviewer and the visible information about a patch under review (e.g., comments and votes that are provided by prior co-reviewers). We then investigate the association between the characterized review dynamics and the defect-proneness of a patch. Through a case study of 83,750 patches of the OpenStack and Qt projects, we observe that the amount of feedback (either votes and comments of prior reviewers) and the co-working frequency of a reviewer with the patch author are highly associated with the likelihood that the reviewer will provide a positive vote to accept a proposed patch. Furthermore, we find that the proportion of reviewers who provided a vote consistent with prior reviewers is significantly associated with the defect-proneness of a patch. However, the associations of these review dynamics are not as strong as the confounding factors (i.e., patch characteristics and overall reviewing activities). Our observations shed light on the implicit influence of the visible information about a patch under review on the evaluation decision of a reviewer. Our findings suggest that the code reviewing policies that are mindful of these practices may help teams improve code review effectiveness. Nonetheless, such review dynamics should not be too concerning in terms of software quality.
\end{abstract}

Index Terms-Code review, Collaboration, Human Aspects, Software Quality, Peer Review, Biases

\section{INTRODUCTION}

Code review is one of the important quality practices in a software development process. Broadly speaking, a proposed patch (i.e., a set of code changes) must be examined and critiqued by team members other than the patch author before their integration into the main software repository. The main goal of code reviews is to improve the overall quality of a patch [4]. Recent work shows that active and rigorous code reviews can improve the quality of system design [48] and decrease the number of post-release defects [5, 45, 63, 72].

Unlike the traditional code review process of the past where reviewers independently examined software artifacts [2. 22], contemporary code review processes often provide a transparent environment with convenient access to reviewer feedback (e.g., comments) in order to enhance active and timely collaboration [4, 17, 56]. Developers in GitHub projects also perceive that such a transparent code review process allows the evaluation decision (i.e., whether or not a new patch should be accepted) to become more democratic [42].

On the other hand, code reviewing is performed by humans who may have subconscious biases that influence their objective evaluation [25]. Prior studies point out that visible information other than the technical content like reviewing discussion may also influence the evaluation decision of code reviews [13, 79, 80]. Tsay et

- P. Thongtanunam is with the University of Melbourne, Australia. E-mail:patanamon.t@unimelb.edu.au.

- A. E. Hassan is with Queen's University, Canada. E-mail:ahmed@cs.queensu.ca. al. observe that core members sometimes use feedback and discussions of other team members to decide whether or not a patch should be accepted [80]. A survey study by Bosu et al. reports that the relationship between a patch author and a reviewer often affects the decision of accepting a review request of the reviewer [13]. A recent study demonstrates that OpenStack developers are starting to perceive unfairness in code reviews [24]. Moreover, Mozilla has recently started an experiment to reduce the gender bias in code reviews [41]. Such biases may potentially affect the long term success of a project.

Several intrinsic biases such as cognitive particularism [78], favouritism for the famliar [53], and peer bias [23] were also identified and discussed in the context of peer reviews for grant proposals. Reviewing rules and approaches were developed in order to mitigate biases [37]. Other reviewing processes like the review of academic publications use anonymization (e.g., doubleblind reviews) to achieve effective reviews [25, 62, 66 , 77]. Yet, little is known whether or not such practices should also be applied to the code review process.

In this study, we perform an empirical study to examine review dynamics (i.e., the practices of making an evaluation decision for a code patch) and their association with the defect-proneness of a patch. In particular, we perform a two-fold analysis to characterize review dynamics (Analysis 1) and investigate the impact of the characterized review dynamics on software quality (Analysis 2). Through a case study of 83,750 patches that spread across the OpenStack and Qt open source projects, we make the following observations.

Analysis 1: Characterizing Review Dynamics. To 
operationalize review dynamics, we investigate the signals of visible information about a patch under review that are associated with the evaluation decision of a reviewer (e.g., whether to vote positively or negatively for a patch). To capture visible information about a patch under review, we use eight metrics that are grouped into three dimensions: (1) feedback during the review (e.g., the prior votes of co-reviewers), (2) status (e.g., whether a co-reviewer is a project core member), and (3) relationship (e.g., the co-working frequency of reviewers). We then use a mixed-effects logistic regression model to estimate the association between these metrics and a positive vote to accept a patch of a reviewer.

Findings: While controlling for several patch characteristics (e.g., patch size) that are known to influence the evaluation decision [26, 32], we find that our newly proposed metrics are associated with the evaluation decision of a reviewer. In particular, our results show that the proportion of positive votes and comments that are provided by prior reviewers are significantly associated with the likelihood that a reviewer will provide a positive vote to accept the patch. We also find that the co-working frequencies with the patch authors are associated with the evaluation decision of a reviewer.

Analysis 2: Investigating the Impact of Review Dynamics on Software Quality. Based on the findings of Analysis 1, we formulate six hypotheses that are related to software quality. For example, we hypothesize that an accepted patch where each of its reviewers provided a positive vote consistent with prior reviewers is more likely to be defective in the future. To test our hypotheses, we define six metrics that are related to the findings of Analysis 1. We then build and examine defect models, i.e., the logistic regression models that estimate the likelihood of a patch inducing fixes.

Findings: While our defect models control for several confounding factors that are known to have an impact on software quality [43], we find that the defectproneness of a patch is associated with the proportion of reviewers who provided a positive vote consistent with prior reviewers and the proportion of reviewers who have a strong relationship with either the patch author or co-reviewers of that patch in OpenStack. Yet, the associations of review dynamics with defect-proneness are not as strong as the patch characteristics and overall reviewing activities.

Our findings shed light on the implicit influence that the visible information in contemporary code review processes may have on the evaluation decision of a reviewer. Such review practices have a relatively small impact on software quality. These findings suggest that the code reviewing policies that are mindful of these practices may help teams improve code review effectiveness. However, teams should not be too concerned about the influence of the visible information on software quality. To facilitate future replication of our study, a replication package of our work is available online ${ }^{1}$

\subsection{Novelty Statement}

This paper is the first to present:

(1) An analysis of the visible information about a patch under review and the evaluation decision of a patch.

(2) An analysis of the association between such visible information and the defect-proneness of a patch.

\subsection{Paper organization}

Section 2 provides a background and a motivation for our work. Section 3 describes the design of our case study. Section 4 presents a preliminary analysis of review dynamics. Sections 5 and 6 present the results of our analyses. Section 7 discusses a broader implication of our results. Section 8 discusses possible threats. Finally, Section 9 draws conclusions.

\section{BACKGROUND \& MOTIVATION}

In this section, we overview contemporary code review processes and motivate our work based on manual observations and related work.

\subsection{Contemporary Code Review Processes}

Code reviews have been widely used in open source and industrial software projects [6, 15, 32, 58,-60]. One of the main motivations for using code reviews is to improve the quality of new patches [4, 13, 59]. Several prior studies show that code reviews impact software quality [5, 45, 63, 72].

In recent years, code review practices have converged to contemporary code reviews which are supported by code review tools [56]. Typically, a code review tool (e.g., Gerrit ${ }^{2}$ ReviewBoard ${ }^{3}$ and Phabricator ${ }^{4}$ is a web-based application that tightly integrates with Version Control Systems (VCSs, e.g., Git). Broadly speaking, the code review process consists of four main steps:

1. The patch author uploads a new patch to the code review tool.

2. Reviewers evaluate the proposed patch and provide feedback. The feedback can be either a review comment or a vote. A positive vote indicates that the patch can be integrated into the upstream VCS, while a negative vote indicates that the patch is not ready for integration.

3. The patch author revises the patch to address the review feedback and uploads a new revision to the code review tool.

4. The review process is repeated until reviewers provide sufficient positive votes indicating that the patch is of sufficient quality to be integrated (i.e., a vote score of +2$)^{5}$

1. https://github.com/SAILResearch/replication_review-dynamics

2. https://www.gerritcodereview.com/

3. https://www.reviewboard.org/

4. http://phabricator.org/

5. https://wiki.qt.io/Qt_Contribution_Guidelines and https://docs. openstack.org/intra/manual/developers.html\#code-review 


\subsection{A Transparent Environment of Code Reviews}

To enable a collaborative code review process, a code review tool often provides transparency in the form of visibility to others' activities on shared artifacts [17, 52. 60. 79]. For example, one can read others' patches and code reviews to learn about other parts of the system without writing code [4]. One also can perceive others' expertise through their visible history of code commits [17]. Developers at Google note that team awareness is one of the motivations of using such transparent code reviews [60.

Unlike traditional code inspections where reviewers have face-to-face meetings [22], contemporary code reviews are performed through a virtual workspace (i.e., the online code review tool). Such a virtual communication and decision making may have an impact on the effectiveness of code reviews [19, 46, 54]. Moreover, based on conventional wisdom, a new patch should be evaluated solely based on technical merits [61]. For example, recent studies observe that in a transparent environment of code reviews, visible information about the author of a patch can affect the evaluation decision [11, 79]. Hence, it is possible that the visible information about co-reviewers may affect the evaluation decision. Below, we discuss possible information that may affect the evaluation decision of a patch based on our manual observations and published literature.

\subsubsection{Manual Observations}

We randomly select 20 patches from the OpenStack project and manually examine their review discussions. Through our manual examination, we observe that for some patches, reviewers provided a vote consistent with prior reviewers and changed their votes based on the review discussion. We provide examples for such two practices below.

Providing Consistent Votes. In the review ID 1411, we observe that although a reviewer has a concern, that reviewer still provides a positive vote based on the vote of prior reviewers: "...It would be nice to fix ..., but that is not urgent for the release. Given that (the prior reviewer) already $+1^{\prime} d$ this patch as well, I will +2 it" 6 . We also observe that reviewers provide a negative vote to support the concerns of prior reviewers. For example, in the review ID 1257, the first reviewer raises a concern. Then, the second reviewer provides a negative vote to support the concern of the first reviewers: "Backing up (the first reviewer)'s comment on....." 7 These examples provide evidence that in addition to the technical merits of a patch, the feedback of prior reviewers influence the evaluation decision of subsequent reviewers.

Changing Votes. In the review ID 344949, we observe that one of the reviewers turns his positive vote to a negative vote after the other co-reviewer raises a concern: "...Code-Review-1. (the co-reviewer)'s right" 8 The

\footnotetext{
6. https://review.openstack.org/\#/c/1411

7. https://review.openstack.org/\#/c/1257

8. https://review.openstack.org/\#/c/344949
}

negative vote of a reviewer can also be turned into a positive vote based on the feedback of other reviewers. For example, in the review ID 16888, a reviewer (R) provides a negative vote and proposes an alternative approach for the patch! ${ }^{0}$ Then, a co-reviewer provides a positive vote to the patch and posts a comment that the patch is acceptable "I think there is some value in $R$ 's proposal, but I don't think it might be a blocker for approval." Finally, the reviewer $\mathrm{R}$ turns his negative vote to a positive vote "After reading the reviews..., I agree with the approach....". These examples provide evidence that the comments of other reviewers may influence the evaluation decision of a reviewer.

\subsubsection{Related Work}

We now discuss other visible information that may also affect an evaluation decision based on literature.

Feedback. While reviewers can discuss concerns and potential defects with other team members in a review discussion thread, such visible feedback (e.g., review votes and comments) may affect the evaluation decision. This influence can be considered as social influence where a popular option tends to continue receiving attention [20, 38, 51]. Prior studies in political science show that the perception around a community's opinion can influence the voting decision of an individual [40, 69]. In the code review context, Tsay et al. point out that team members sometimes apply pressure during a review discussion to influence the patch acceptance of a core member [80].

Status. Intuitively, the longer the provided review feedback, the more effective the code review is [36]. However, several studies report that not every feedback will receive attention during the code review [9, 59, 80]. For example, Rigby et al. observe that the feedback of outsiders (e.g., non-core members) tends to receive less attention than that of core members during code reviews [59]. This can be considered as a cognitive bias in terms of social hierarchy, where an implicit or explicit rank order of individuals or group influence others' behaviors [14, 38]. Studies of peer reviews in academic publications point out that the reputation of authors can influence reviewers [27, 77]. In the code review context, a recent study observes that the social status of a patch author (e.g., the number of followers in GitHub projects) is associated with the likelihood of patch acceptance [79]. Bosu et al. report that in open source projects, developers' reputation (based on social network analysis) can influence the evaluation decision of their patches [11].

Relationship. Since team members often interact with each other during the code review process, they may form these interactions into a relationship. Then, it is possible that a reviewer may trust the contributions (either a patch or review feedback) of team members with whom they have a strong relationship. Such a behavior can be considered as a type of ingroup-outgroup

9. https://review.openstack.org/\#/c/16888 
behavior, where individuals create a strong motivation to cooperate with ingroup members [38]. For example, a study of the peer review of academic publications found that the publications of newcomers are underrepresented when revealing the author names [62]. Similarly, due to the social dynamics in code reviews, the collective evaluation of a patch acceptance can be affected by the interpersonal compatibility of reviewers [1, 49]. In the context of code reviews, a recent study also reports that the relationship with the patch author is one of the important factors for open source developers to decide whether they will review a patch [13]. On the other hands, studies in open source projects report that developers with a weak relationship with other team members like newcomers face negative impressions (e.g., receiving slow feedback) from reviewers [39, 68].

\section{Case Study Design}

In this section, we provide an overview of our study, the studied projects, our data preparation, and our employed statistical analysis approaches.

\subsection{Study Overview}

To better understand the review dynamics of modern transparent code review processes and the impact of such dynamics on software quality, we perform an empirical study using a two-fold analysis. Figure 1 provides an overview of our case study design, which we briefly describe blow.

\section{Analysis 1: Characterizing Review Dynamics}

To operationalize review dynamics, we analyze historical data to identify signals that can relate to the evaluation decision of each reviewer in each patch using a mixedeffects logistic regression model. In particular, for each patch, we extract the visible information available during its code review using eight metrics grouped along three dimensions, i.e., feedback during reviews, status, and relationship. Then, we examine the association between these metrics and the likelihood that a reviewer will provide a positive vote to a patch.

\section{Analysis 2: The Impact of Review Dynamics on Software Quality}

Once we identify important review dynamics, i.e., significant signals of visible information metrics to an evaluation decision, we examine whether such review dynamics have an impact on software quality. To do so, we formulate hypotheses based on the findings of Analysis 1 and develop corresponding metrics for each of our hypotheses. Then, we analyze defect models to examine the association between our review dynamics metrics and the likelihood that a patch will be defective.

\subsection{Studied Projects}

To understand the review dynamics of the modern code review process, we perform an empirical study on large open source projects that actively use code reviews. To select the studied projects, we start with the open source projects that are listed in the work of Bosu and Carver [11]. Then, we check for the accessibility of the data of the code review tool and the VCS of these projects. For example, the ITK/VTK project was not included in our study because its REST API access is no longer provided.

We obtain OpenStack, Qt, LibreOffice, and Chromium. These four projects have a large number of reviews recorded in the Gerrit code review tool (see Table 1). However, we observe that a large proportion of LibreOffice reviews have only one reviewer. Since we want to investigate the review dynamics and interactions among reviewers during a code review, we exclude LibreOffice out from our study. Similarly, we observe that Chromium reviewers rarely use a negative vote for both merged and abandoned patches (see Table 1 in the \#Patches with +vote/-vote column). Since our Analysis 1 investigates the signals that can relate to the evaluation decision (i.e., providing a positive or negative vote), we exclude Chromium from our study. Therefore, in this paper, we perform an empirical study on the OpenStack and Qt projects.

The OpenStack project is managed by the OpenStack Foundation with code contributions from well-known companies (e.g., IBM, EMC, Cisco) ${ }^{10}$ Qt was mainly developed by the Qt company. Currently, the Qt project is led by the Nokia and Digia corporation, nevertheless, code contributions from the community are also welcomed11

\subsection{Data Preparation}

Below, we describe the data extraction and cleaning approaches.

Data Extraction. We collect the review data from the Gerrit tool of the OpenStack ${ }^{12}$ and $Q^{\sqrt{13}}$ projects using the REST API. Our review datasets include review ID, patch information (e.g., patch description, modified files), revisions, review discussion threads, and the involved personnel that are recorded in the tool. Then, we measure the overall review activities which are described in Table 2 .

We also extract the score of each vote and its timestamps for our Analysis 1. To do so, we use a set of regular expressions to extract the votes from the review messages in the discussion threads. The timestamps when the corresponding message was posted is considered as the time when the vote was provided. We can rely on this approach since Gerrit automatically records

10. https://www.openstack.org/

11. https://www.qt.io/developers/

12. https://review.opendev.org/

13. https://codereview.qt-project.org/ 


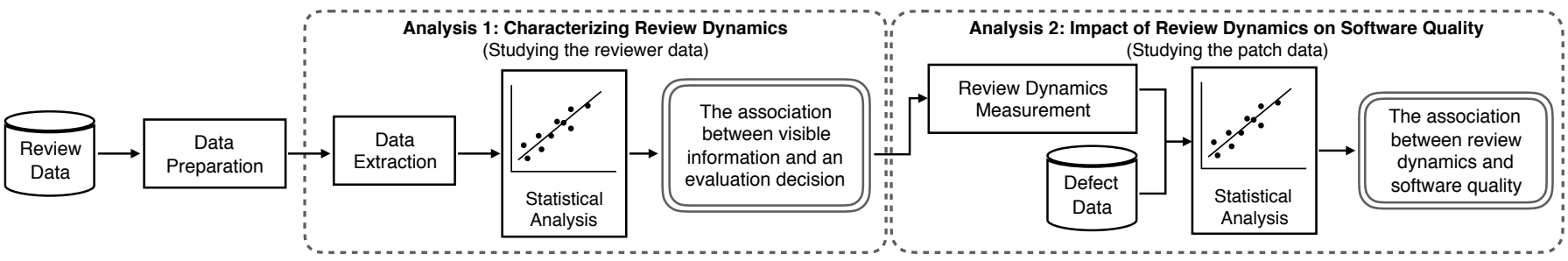

Fig. 1: An overview of the design of our case study.

TABLE 1: An overview of the review datasets.

\begin{tabular}{|c|c|c|c|c|c|c|c|}
\hline & Period & \#Patches & \#Reviewers & \#Reviewers/Patch & $\begin{array}{c}\text { \#Patches w/ } \\
>1 \text { Reviewers }\end{array}$ & $\begin{array}{l}\text { \#Patches w/ } \\
\text { +Vote/-Vote }\end{array}$ & $\begin{array}{r}\text { \#Commits } \\
\text { (\%FixInducing) }\end{array}$ \\
\hline OpenStack & $11 / 2011-07 /$ & 782 & 3,662 & Avg $=6(\min =1, \max =43)$ & $57,288(99 \%)$ & $50,374 / 32,074$ & $35,885(49 \%)$ \\
\hline Qt & 0 & & 694 & Avg $=2$ & $15,765(60 \%)$ & 3,626 & 18,88 \\
\hline Libre & $07 / 2012-06 /$ & $\overline{4}$ & 24 & Avg $=1$ & $3,639(9 \%)$ & $16,248 / 2,490$ & - \\
\hline
\end{tabular}

the history (e.g., a vote score and patch revision) in the discussion threads ${ }^{14}$ The first line of a review message will contain the vote score following specific patterns (e.g., 'Patchset 1: Code-Review $+1^{\prime}$ ) when a reviewer provides a vote. We also manually validate the extraction results.

For the code datasets, we use PyDriller, a Python framework [65], to collect code commits from the Git VCSs of the studied projects and extract code characteristics which are described in Table2 2 Then, we use the SZZ algorithm [64] to identify fix-inducing commits. To do so, we identify fixing commits using keyword search (e.g., fix, bug, and defect). Then, we use the implementation of Pydriller to identify a set of the commits that recently modified the same lines in the files that are included in the commit of interest 15 Finally, those recent commits that made changes to the same lines prior to the commit of interest are identified as fix-inducing commits.

Once we collect the review and code datasets, we link the data using the review ID. Review ID is a unique identifier that follows the Project Branch Change-ID format, where Project is the name of the VCS repository, Branch is the destination branch into which the patch will be merged, and Change-ID is a 41-digits hash value. According to the contribution guideline of the studied projects ${ }^{16}$ the Change-ID is automatically generated and inserted into the commit message when the proposed patch is merged into the main VCS. Hence, we use a regular expression to extract the Change-ID in the commit message. Then, we use the extracted Change-ID of a commit and its corresponding VCS repository and branch to link between the code commits and the reviews in our datasets. Note that in this work, we study only commits that are merged into the main branch.

14. https://gerrit-review.googlesource.com/Documentation/ user-review-ui.html\#history

15. https://pydriller.readthedocs.io/en/latest/reference.html\# pydriller.git_repository.GitRepository.get_commits_last_moditied_ lines

16. https://docs.openstack.org/infra/manual/developers.html
Data Cleaning. To accurately understand review dynamics, we clean the studied review datasets by merging duplicate accounts due to email aliases [9]. To do so, we search for a pair of accounts that share the same substring of the name or the email name (excluding the email domain). We then manually verify each pair of potential duplicates. In addition, we remove the messages that are posted by automated tools in a review discussion thread [76]. To do so, we study the documentation of the studied projects ${ }^{17}$ to identify the automated tools that are integrated with the code review tools.

Table 1 provides a summary of the review and code datasets that will be used in this study. The number of patches column indicates the number of patches that are marked as merged or abandoned. We only consider the proposed patches that were submitted after November 2011 for OpenStack and September 2013 for Qt since prior work pointed out that the earlier period may be the initial adoption period of code reviews in those projects [43].

\subsection{Statistical Analysis}

To statistically analyze the data, we use a regression model to fit our data while controlling for several confounding factors. Similar to prior studies [10, 36, 79, 81], our main goal of using regression models is not for prediction, but to understand the associations between the metrics of interest and the likelihood that is estimated by the regression models [70].

Confounding factors. Table 2 briefly describes the 22 metrics that we used as confounding factors. For both Analysis 1 and Analysis 2, we use 15 code metrics as confounding factors in terms of patch characteristics. These code metrics are commonly known to have an association with defect-proneness [10, 18, 30, 34, 43, 55, 73]. We also consider the description length of a patch as a confounding factor since our prior work finds that the

17. https://docs.openstack.org/infra/manual/developers.html and https://wiki.qt.io/CI_Overview 
TABLE 2: An overview of confounding factors.

\begin{tabular}{|c|c|}
\hline \multicolumn{2}{|l|}{ Patch characteristics } \\
\hline Metric & Description \\
\hline \#Added lines & The number of lines that were added by the studied patch. \\
\hline \#Deleted lines & The number of lines that were deleted by the studied patch. \\
\hline LOC & The number of lines of code in the files before the change by the studied patch. \\
\hline Average Complexity & The average Cyclomatic Complexity of the files that were impacted by the studied patch. \\
\hline \#Files & The number of files that were impacted by the studied patch. \\
\hline \#Directories & The number of directories that were impacted by the studied patch. \\
\hline Is bug fixing & Whether or not the studied patch is for fixing bugs. \\
\hline Entropy & The dispersion of modified lines across files $[30]$. \\
\hline \#Developers & $\begin{array}{l}\text { The number of developers who made prior patches that impact the same modified files as the studied } \\
\text { patch. }\end{array}$ \\
\hline \#Prior patches & The number of prior patches that impact the same modified files as the studied patch. \\
\hline \#Prior fixes & $\begin{array}{l}\text { The number of prior patches that are for bug fixing and that impact the same modified files as the } \\
\text { studied patch. }\end{array}$ \\
\hline Age & $\begin{array}{l}\text { The average of the time intervals between the last patch that was made to each modified file and } \\
\text { the studied patch. }\end{array}$ \\
\hline Is the patch author major author & $\begin{array}{l}\text { Whether the authoring-specific ownership of the patch author is greater than } 0.05 \text {, where the } \\
\text { authoring-specific ownership is the proportion of prior patches that are made by the patch author, } \\
\text { and that impacted the same modified files of the studied patch [10]. }\end{array}$ \\
\hline Is the patch author major reviewer & $\begin{array}{l}\text { Whether or not the review-specific ownership of the patch author is greater than } 0.05 \text {, where the } \\
\text { review-specific ownership is the proportion of prior patches that are reviewed by the patch author, } \\
\text { and that impacted the same files as the modified files of the studied patch [73]. }\end{array}$ \\
\hline Patch description length & Word count of the commit message that the patch author used to describe the studied patch [74]. \\
\hline \multicolumn{2}{|l|}{ Reviewer Characteristics } \\
\hline Metric & Description \\
\hline Review involvement & $\begin{array}{l}\text { The proportion of comments that the reviewer under study provides to the studied patch before the } \\
\text { reviewer under study provides a vote [73] } 74] \text {. }\end{array}$ \\
\hline Reviewer authoring experience & $\begin{array}{l}\text { The proportion of prior patches that are made by the reviewer, and that impacted at least one of the } \\
\text { modified files of the studied patch [10]. }\end{array}$ \\
\hline Reviewer reviewing experience & $\begin{array}{l}\text { The proportion of prior patches that are reviewed by the reviewer under study, and that impacted } \\
\text { at least one of the modified files of the studied patch [73]. }\end{array}$ \\
\hline \multicolumn{2}{|l|}{ Reviewing activities } \\
\hline Metric & Description \\
\hline \#Reviewers & The total number of reviewers who provided a vote for the studied patch [72]. \\
\hline \#Reviewing comments & The total number of comments of reviewers [44 [72] 74]. \\
\hline \%Positive votes & The proportion of reviewers who provided a positive vote for the studied patch [72] 74$]$. \\
\hline Reviewing time & $\begin{array}{l}\text { The length of time between the patch submission to the code review tool and the final evaluation } \\
\text { outcome [72] } 74] \text {. }\end{array}$ \\
\hline
\end{tabular}

description length is associated with the participation of reviewers [74].

In addition, in Analysis 1, we also consider the past involvement and experience of reviewers. This is because prior work shows that the review quality can be associated with the reviewer experience [36, 57, 73]. We use three metrics to measure the characteristics of a reviewer (see Table 2).

Since overall reviewing activities also can have an impact on software quality [5, 36, 45, 72], we also use four code review metrics as confounding factors for Analysis 2 (see Table 2). These code review metrics measure the reviewing activities that occur during the review until the evaluation decision was made.

Our statistical analysis approach consists of four main steps, which we describe in detail below.

\subsubsection{Correlation Analysis}

A high correlation among metrics may lead a regression model to produce spurious associations between the metrics and the estimated likelihood [29, 70]. Hence, we use Spearman rank correlation $(\rho)$ to assess the correlation between each pair of metrics. For each pair of metrics with a correlation $|\rho|$ larger than 0.7 , we remove one metric from our study. To systematically and consistently select metrics for our study, we use the Autospearman function in the Rnalytica $R$ package to find the metric that has the most unique signal, i.e., having the least correlation with other metrics in the dataset, without considering the response variable [33].

\subsubsection{Fitting Statistical Models}

We now describe our approach of fitting the regression models for Analysis 1 and 2 (see Figure 1).

Review Dynamics Models. In our Analysis 1, we analyze the association between the visible information about a patch under review and the evaluation decision of a reviewer (i.e., whether a reviewer provides a positive vote to accept a patch). Since the evaluation decision of each reviewer is dependent on the actual patch, we use a mixed-effects logistic regression model to account for variance in the multilevel data (i.e., the reviewer evaluation decision and the patch data) [67]. We use the glmer function in the lme $4 \mathrm{R}$ package with the option of family='binomial' to fit mixed-effects logistic regression models to our data.

Defect Models. In our Analysis 2, we analyze how review dynamics in a patch are associated with defect- 


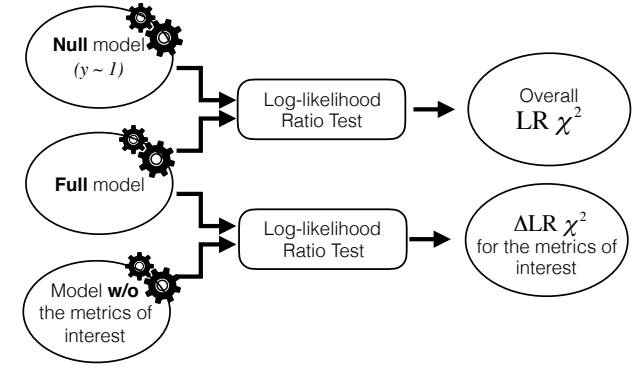

Fig. 2: An overview of log-likelihood ratio (LR) tests.

proneness. To do, we use a simple logistic regression model to fit our data. We use the glm function in the stats $R$ package with the option of family='binomial'.

\subsubsection{Assessing the Fit}

To assess the goodness of fit, we use a log-likelihood ratio test [31] and the Area Under the receiver operating characteristic Curve (AUC) [28].

Log-likelihood ratio tests. We use a log-likelihood ratio (LR) test to evaluate how well our regression models fit to the data using the studied metrics. Figure 2 provides an overview of our LR tests.

1) Overall $L R \chi^{2}$ indicates the overall goodness of fit for the model that uses all metrics (called a full model). To estimate the overall $\mathrm{LR} \chi^{2}$, we compare our full model against the null model (i.e., a model that fits the data without metrics except an intercept).

2) $\Delta L R \chi^{2}$ indicates the goodness of fit for the metrics of interest in the model. To estimate the $\Delta \mathrm{LR} \chi^{2}$, we compare the full model against the model that fits the data without using the metrics of interest.

The larger the overall $\mathrm{LR} \chi^{2}$ (or $\Delta \mathrm{LR} \chi^{2}$ ) value is, the better the fit of our model based on all metrics (or a particular set of metrics).

AUC. We use AUC to evaluate how well a model can discriminate between two potential responses. We use the auc function in the $P R O C R$ package to measure the AUC for our models. An AUC value of 1 indicates the best discriminant ability of the model, while an AUC value of 0.5 indicates that the discriminant ability of the model is no better than random guessing.

Validating the models. We use the bootstrap validation technique [21] to estimate the optimism of AUC. As suggested by prior work [71], the bootstrap validation technique tends to yield less bias than the traditional $\mathrm{k}$-fold cross validation techniques for logistic regression models. To estimate the AUC optimism, we first generate a bootstrap sample, i.e., a sample with replacement of the studied dataset. Then, we build a model using the bootstrap sample (i.e., a bootstrap model). Finally, the AUC optimism is the difference in AUC between the bootstrap model when applied to the original dataset and the bootstrap sample. We repeat this process 100 times and compute the average AUC optimism. The

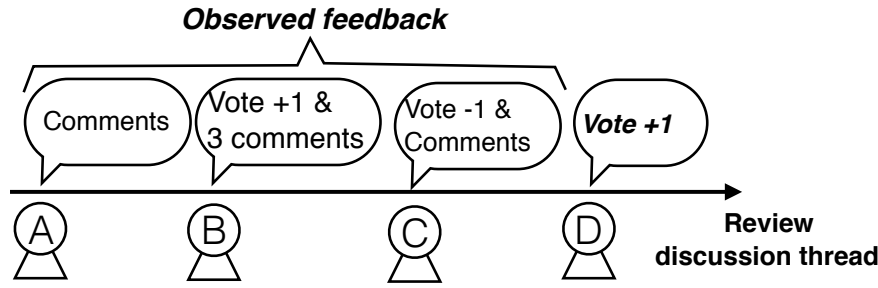

Fig. 3: An example of prior visible feedback that are used to compute metrics.

small AUC optimism indicates that the performance estimates of our models using the original data are reliable.

\subsubsection{Analyzing Associations}

We use Wald statistics to estimate the explanatory power of each metric that contributes to the fit of the regression model. To do so, we use the Anova function in the car $\mathrm{R}$ package with the option of test. statistic $=$ 'Chisq' to estimate the explanatory power (Wald $\chi^{2}$ ) and its statistical significance ( $p$-value). The larger the Wald $\chi^{2}$ value is, the larger the explanatory power that a particular metric contributes to the model. In addition, we examine the direction of the association by observing the regression coefficients of the regression model.

\section{Preliminary Analysis}

In Section 2.2.1. we observe that for some patches, reviewers provided a vote consistent with prior reviewers and changed their votes based on the review discussion. This observation provides evidence that feedback of other prior reviewers influences the evaluation decision of a reviewer. Therefore, we perform a preliminary analysis to examine whether such practices are associated with defect-proneness.

Approach. We first measure the proportion of reviewers who provide a positive vote consistent with prior reviewers and the proportion of reviewers who change their vote on the same patch revision. For our calculation, we assume that reviewers may observe all the visible information when making an evaluation decision. For example, in Figure 3, reviewer D may observe the visible information, i.e., the prior feedback of reviewers $\mathrm{A}, \mathrm{B}$, and C; and provide a vote consistent with a prior reviewer (i.e., reviewer B). Therefore, the proportion of reviewers who provide a consistent positive vote for this review is $\frac{1}{3}$. In this analysis, we did not measure the proportion of reviewers who provide a consistent negative vote, since in Section 2.2.1. we observe that reviewers provide a positive vote consistent with the prior reviewers.

Unlike the proportion of positive votes in the overall review activities (see Table 2) which measures the consensus at the time when the patch was accepted, the proportion of reviewers who provided a vote consistent 
TABLE 3: Statistics of defect models with the observed review dynamics. The larger the Wald $\chi^{2}$ value of a metric is, the larger the explanatory power of this particular metric in the model.

\begin{tabular}{|c|c|c|}
\hline & OpenStack & $\overline{Q t}$ \\
\hline \#TRUE/FALSE Instances & $17,924 / 17,962$ & $4,857 / 14,030$ \\
\hline Overall LR $\chi^{2}$ & $6,677^{* * *}$ & $1,919^{* * *}$ \\
\hline AUC & 0.74 & 0.70 \\
\hline Review Dynamics & Wald $\chi^{2}$ & Wald $\chi^{2}$ \\
\hline \%Consistent with prior positive votes & $14^{* *}(+)$ & $0 \circ(+)$ \\
\hline$\%$ Vote changes & $0 \circ(-)$ & $5^{*}(-)$ \\
\hline Reviewing Activities & Wald $\chi^{2}$ & Wald $\chi^{2}$ \\
\hline \%Positive votes & $180^{* * *}(-)$ & $167^{* * *}(-)$ \\
\hline \#Reviewing comments & $736^{* * *}(+)$ & $147^{* * *}(+)$ \\
\hline Reviewing time & $188^{* * *}(-)$ & $8 * *(+)$ \\
\hline Patch Characteristics & Wald $\chi^{2}$ & Wald $\chi^{2}$ \\
\hline \#Added lines & $14^{* * *}(+)$ & $10(+)$ \\
\hline \#Deleted lines & $1 \circ(-)$ & $34^{* * *}(+)$ \\
\hline LOC & $176^{* * *}(-)$ & $\dagger$ \\
\hline Avg. complexity & $143^{* * *}(-)$ & $42 * * *(-)$ \\
\hline \#Directories & $168^{* * *}(+)$ & $4^{*}(-)$ \\
\hline Is Bug Fixing & $328 * * *(+)$ & $8^{* *}(+)$ \\
\hline Entropy & $309 * * *(+)$ & $174^{* * *}(+)$ \\
\hline \#Prior fixes & $561 * * *(+)$ & $249^{* * *}(+)$ \\
\hline Age & $130^{* * *}(-)$ & $32 * * *(-)$ \\
\hline Is the patch author major author & $100^{* * *}(+)$ & $68^{* * *}(+)$ \\
\hline Is the patch author major reviewer & $0 \circ(-)$ & $3 \circ(+)$ \\
\hline \multicolumn{3}{|c|}{$\begin{array}{l}\text { Stat. significance: }{ }^{* * *} p<0.001,{ }^{* *} p<0.01,{ }^{*} p<0.05,{ }^{\circ} p \geq 0.05 \\
\dagger \text { Discarded due to the correlation analysis. \#Reviewers, } \\
\text { \#Prior patches, \#Developers, \#Files are also discarded due to the correlation } \\
\text { analysis. }\end{array}$} \\
\hline
\end{tabular}

with prior positive votes does not measure the proportion of the reviewers at the time when the patch was accepted. Indeed, our new metric measures the proportion of reviewers at the time when each reviewer in a review provided a vote. Hence, the proportion of reviewers who provided a vote consistent with prior positive votes can have a different value from the proportion of positive votes. Finally, we examine the association between these metrics and the likelihood of a patch inducing fixes using defect models. We construct our defect models as described in Section 3.4

Preliminary Findings. Table 3 shows that there is a significant positive association between the proportion of reviewers who provide a consistent positive vote with prior reviewers on the same patch (i.e., the proportion of consistent positive votes) and the likelihood of inducing fixes for OpenStack dataset. This result indicates that the proportion of consistent votes is associated with defectproneness even when reviewing and code confounding factors are controlled. More specifically, although we control for overall positive votes (i.e., the proportion of positive votes) in our defect models, the explanatory power (Wald $\chi^{2}$ ) of the proportion of consistent votes still accounts for $7 \%\left(\frac{14}{180}\right)$ for consistent positive votes of the explanatory power that the proportion of positive votes contributes to the OpenStack model.

Although the proportion of providing consistent votes did not contribute a significant amount of explanatory power to the Qt model, other visible information about a patch under review may play a role. For example, Table 3 shows that the number of reviewing comments plays a significant role in our defect models. It is possible that the comments of prior reviewers may also influence the evaluation decision of a reviewer. Therefore, we further investigate the review dynamics of code reviews and their association with defect-proneness in the following two sections.

Summary: Despite conventional wisdom [61], our preliminary analysis shows that the visible information in a review discussion might influence the evaluation decision of a review. Such a practice is also associated with defect-proneness for the OpenStack dataset. Yet, review dynamics still remain largely unexplored.

\section{Analysis 1: Characterizing Review DYNAMICS}

In this section, we present the approach and the results of our Analysis 1.

\subsection{Approach}

To examine the review dynamics (i.e., practices of voting for a patch of a reviewer), we compute metrics that capture visible information about a patch under review. Based on literature and our manual observations (see Section 2.2 , we use eight metrics which are grouped into (1) feedback, (2) status, and (3) relationship dimensions. Table 4 describes our seven metrics. Note that in the calculation of these metrics, we use only the information (i.e., votes and comments) that occurs before the reviewer under study provides a vote. For example, given that reviewer D in Figure 3 is the reviewer under study, we use only votes and comments that are provided by the prior reviewers (i.e., reviewers $\mathrm{A}, \mathrm{B}$, and $\mathrm{C}$ ) to compute metrics for reviewer D.

We now build models to analyze the associations between the visible information about a patch under review and the evaluation decision of each reviewer of that patch. In Analysis 1, we perform a study on both merged and abandoned reviews. Since the evaluation decision of each reviewer is dependent on the actual patch, we use a mixed-effects logistic regression model to account for variance in the multilevel data (i.e., the reviewer evaluation decision and the patch data) [67]. We use our metrics that capture the visible information about a patch under review and confounding factors as fixed effects while the unique reviewer ID is represented as a random effect. Since we analyze the activities during a code review, we use only the patch characteristics described in Table 2 as confounding factors. We set the response variable in our models as TRUE if the reviewer under study provides a positive vote to a patch, and FALSE otherwise. The formula used in our mixed-effect models is IsPositiveV ote $\sim x_{1}+x_{2}+\ldots . . x_{n}+(1 \mid$ ReviewerId $)$, where $x_{1}, \ldots, x_{n}$ are the fixed effects (i.e., our studied

18. The core members are identified based on the project documentation, i.e., https://wiki.openstack.org/wiki/Project_Resources and https://wiki.qt.io/Maintainers 
TABLE 4: An overview of our proposed metrics to capture visible information about a patch under review. Note that we only use the information (e.g., votes and comments) that is available before a reviewer under study provides a vote.

\begin{tabular}{|c|c|}
\hline Metric & Description \\
\hline \%Prior negative votes & The proportion of negative votes provided by prior reviewers. \\
\hline$\%$ Prior comments & $\begin{array}{l}\text { The proportion of prior reviewer comments that were posted by prior reviewers } \\
\text { for the recent patch revision relative to the comments for all the patch revisions. }\end{array}$ \\
\hline \multicolumn{2}{|c|}{ Status dimension captures reviewing activities of core members before the reviewer under study provides a vote. } \\
\hline \%Prior positive vote from core reviewers & The proportion of positive votes provided by documented core members ${ }^{18}$ \\
\hline Is the patch author a core member & TRUE if the patch author is a documented core member. ${ }^{11}$ \\
\hline$\%$ Reviewed patches for the patch author & $\begin{array}{l}\text { The proportion between the number of prior patches that the reviewer under } \\
\text { study has reviewed for the patch author and the number of prior patches } \\
\text { reviewed by the reviewer under study. }\end{array}$ \\
\hline $\begin{array}{l}\text { Avg. co-working frequency with prior positive co- } \\
\text { reviewers }\end{array}$ & $\begin{array}{l}\text { An average of the number of prior patches that the reviewer under study had } \\
\text { co-reviewed with each prior positive co-reviewer normalized by the number of } \\
\text { prior patches reviewed by the reviewer under study. }\end{array}$ \\
\hline
\end{tabular}

metrics and confounding factors). Since reviewers can provide a vote to a patch several times, we focus only on their latest vote. Finally, we build and analyze our mixed-effects models (see Section 3.4).

\subsection{Results}

Table 5 shows that our mixed-effects models achieve an AUC of 0.80 and 0.76 and a statistically significant overall $\mathrm{LR} \chi^{2}$ improvement over a null model for both the OpenStack and Qt datasets. Our models also achieve a very small AUC optimism value, indicating that our models achieve a good and stable fit to the data.

Visible information about a patch under review has a stronger association with the evaluation decision than patch characteristics. Table 5 shows that 9 out of the 11 metrics that capture the visible information about a patch under review contribute a significant amount of explanatory power to our models. Moreover, Table 5 shows that the $\Delta \mathrm{LR} \chi^{2}$ values for our studied metrics account for $63 \%$ and $50 \%$ of the overall $\mathrm{LR} \chi^{2}$ in the OpenStack and Qt models, respectively. On the other hand, the $\Delta \mathrm{LR} \chi^{2}$ values of the patch characteristics only account for $15 \%$ and $26 \%$ of the overall $\mathrm{LR} \chi^{2}$. This result indicates that our proposed metrics that capture the visible information about a patch under review are highly associated with the evaluation decision of a reviewer in our models. This result suggests that despite the patch characteristics for which a reviewer should be concerned, the evaluation decision of a reviewer tends to be more related to the visible information in a code review tool.

The relationship with the patch author is highly associated with the evaluation decision of a reviewer. Table 5 shows that there is a significant positive association between the proportion of reviewed patches for the patch author and the likelihood of providing a positive vote by a reviewer. In particular, the proportion of reviewed patches for the patch author contributes a relatively large amount of explanatory power which accounts for $19 \%\left(\frac{2022}{10713}\right)$ and $16 \%\left(\frac{107}{690}\right)$ of the overall $\mathrm{LR} \chi^{2}$ in the OpenStack and Qt models, respectively. This result indicates that the more prior patches that the reviewer under study had reviewed for the patch author in the past, the more likely that the reviewer will provide a positive vote to the patch under review.

The proportion of prior positive votes is significantly associated with the evaluation decision of a reviewer. Table 5 shows that the explanatory power of the proportion of prior positive votes accounts for $15 \%\left(\frac{1658}{10713}\right)$ and $2 \%\left(\frac{12}{690}\right)$ of the overall $\mathrm{LR} \chi^{2}$ in the OpenStack and Qt models, respectively. Furthermore, the proportion of prior positive votes has a positive association with the the likelihood of providing a positive vote by a reviewer, indicating that the more co-reviewers who provided positive votes before the reviewer under study provides a vote, the more likely that the reviewer under study will provide a positive vote to the patch under review.

Similarly, the proportion of prior negative votes contributes a significant amount of explanatory power to the Qt model. The negative association indicates that the reviewer under study is more likely to provide a negative vote when more co-reviewers provided a negative vote.

The proportion of prior reviewer comments is significantly associated with the evaluation decision of a reviewer. Table 5 shows that the proportion of prior reviewer comments contributes a relatively large amount of explanatory power to the OpenStack and Qt models. Table 5 also shows that the proportion of prior reviewer comments has a negative association with the likelihood of providing a positive vote by a reviewer. This result indicates that the more comments that were provided by co-reviewers to the recent patch revision before the 
TABLE 5: Statistics of mixed-effects models. The goodness of fit for the metrics of interest $\left(\Delta \operatorname{LR} \chi^{2}\right)$ is shown in a proportion to the Overall $\mathrm{LR} \chi^{2}$.

\begin{tabular}{|c|c|c|c|}
\hline & & OpenStack & $\mathbf{Q t}$ \\
\hline Response Variable (\#TRUE/FALSE) & & $198,314 / 61,275$ & $36,867 / 4,394$ \\
\hline Overall $\mathrm{LR} \chi^{2}$ & & $10,713^{* * *}$ & $690^{* * *}$ \\
\hline AUC (|Optimsm $\mid)$ & & $0.8(0.005)$ & $0.76(0.011)$ \\
\hline Variance of Random effect & & 1.07 & 0.75 \\
\hline Intercept & $\chi^{2}$ & $2765^{* * *}$ & $918^{* * *}$ \\
\hline (Dimension) Visible information about a patch under review & $\Delta \mathrm{LR}^{2}$ & $6,800^{* * *}(63 \%)$ & $345^{* * *}(50 \%)$ \\
\hline (Feedback) \%Positive votes & $\chi^{2}$ & $1,658^{* * *}(+)$ & $12^{* * *}(+)$ \\
\hline (Feedback) \%Negative votes & $\chi^{2}$ & $\dagger$ & $59^{* * *}(-)$ \\
\hline (Feedback) \%Prior comments & $\chi^{2}$ & $473^{* * *}(-)$ & $32 * * *(-)$ \\
\hline (Status) \%Prior positive votes of core developers & $\chi^{2}$ & $121^{* * *}(+)$ & $0 \circ(+)$ \\
\hline (Status) Is the patch author a core member & $\chi^{2}$ & $609^{* * *}(+)$ & $0 \circ(+)$ \\
\hline (Relationship) \%Reviewed patches for the patch author & $\chi^{2}$ & $2,022 * * *(+)$ & $107^{* * *}(+)$ \\
\hline (Relationship) Avg. co-working frequency with prior positive co-reviewers & $\chi^{2}$ & $\dagger$ & $\dagger$ \\
\hline (Relationship) Avg. agreement level with prior positive co-reviewers & $\chi^{2}$ & $\dagger$ & $\dagger$ \\
\hline Patch Characteristics & $\Delta \mathrm{LR} \chi^{2}$ & $654^{* * *}(15 \%)$ & $180^{* * *}(26 \%)$ \\
\hline \#Added Lines & $\chi^{2}$ & $53^{* * *}(-)$ & $4 \circ(-)$ \\
\hline \#Modified Directories & $\chi^{2}$ & $\dagger$ & $2 \circ(-)$ \\
\hline Entropy & $\chi^{2}$ & $750^{* * *}(-)$ & $90 * * *(-)$ \\
\hline Is the patch author major author & $\chi^{2}$ & $260^{* * *}(+)$ & $53^{* * *}(+)$ \\
\hline Is the patch author major reviewer & $\chi^{2}$ & $146^{* * *}(+)$ & $8^{* *}(+)$ \\
\hline Patch description length & $\chi^{2}$ & $448^{* * *}(-)$ & $19^{* * *}(-)$ \\
\hline
\end{tabular}

reviewer under study provides a vote, the less likely that the reviewer will provide a positive vote to the patch under review.

In the OpenStack dataset, the core member status also contributes explanatory power to the evaluation decision of a reviewer. Table 5 shows that the proportion of prior positive vote from core members and the core member status of the patch author contribute a significant amount of explanatory power to the OpenStack models. This result suggests that in OpenStack, the evaluation decision of a reviewer may also relate to the core member status of prior reviewers and the patch author.

\subsection{Review Dynamics and Reviewer Characteristics}

Although we find that our metrics that capture the visible information about a patch under review have a significant association with the evaluation decision of a reviewer, such an association may not apply to all reviewers. For example, expert reviewers may be more able to identify a hidden problem in a patch than novice reviewers [36, 57, 73]. Hence, we further investigate whether reviewer characteristics influence the associations between our metrics that capture visible information about a patch under review and the evaluation decision of a reviewer.

Similar to prior work [79], we add an interaction term between the metrics of interest (i.e., the proportion of prior positive votes, prior reviewer comments, and reviewed patches for the patch author) and reviewer characteristics into our mixed-effects models. We use three metrics that we use to measure reviewer characteristics (see Table 2). We then refit and analyze our mixedeffects logistic regression models.

We find that the overall $\mathrm{LR} \chi^{2}$ values of our new mixed-effects models that include reviewer charac- teristics and their interaction terms decrease by $6 \%$ $\left(\frac{(10021-10713)}{10713}\right.$ for OpenStack) and increase by $13 \%$ $\left(\frac{(784-690)}{600}\right.$ for $\left.\mathrm{Qt}\right)$ from the original models in Table 5 However, the AUC values remain the same as the original mixed-effects models. These results indicate reviewer characteristics have a relatively small impact on the association between the visible information about a patch under review and the evaluation decision of a reviewer.

Summary: Visible information about a patch under review has a significant association with the evaluation decision. The proportion of prior positive votes, prior reviewer comments, and the proportion of reviewed patches for the patch author share a significant association with the evaluation decision of a reviewer.

\section{Analysis 2: Impact of ReView Dynam- ICS ON SOFTWARE QUALITY}

The results of Analysis 1 show that our metrics that capture the visible information about a patch under review (i.e., feedback and relationship with the patch author) are highly associated with the evaluation decision of a reviewer. For example, we find that the more co-reviewers who provided positive votes before the reviewer under study provides a vote, the more likely that the reviewer under study will provide a positive vote to the patch under review. Yet, little is known about the risk that such review dynamics (i.e., a practice of providing an evaluation decision by a reviewer in a code review) can have on software quality. Hence, we set out to investigate the relationship between the review dynamics and defect-proneness. 
TABLE 6: An overview of metrics for measuring review dynamics in a patch.

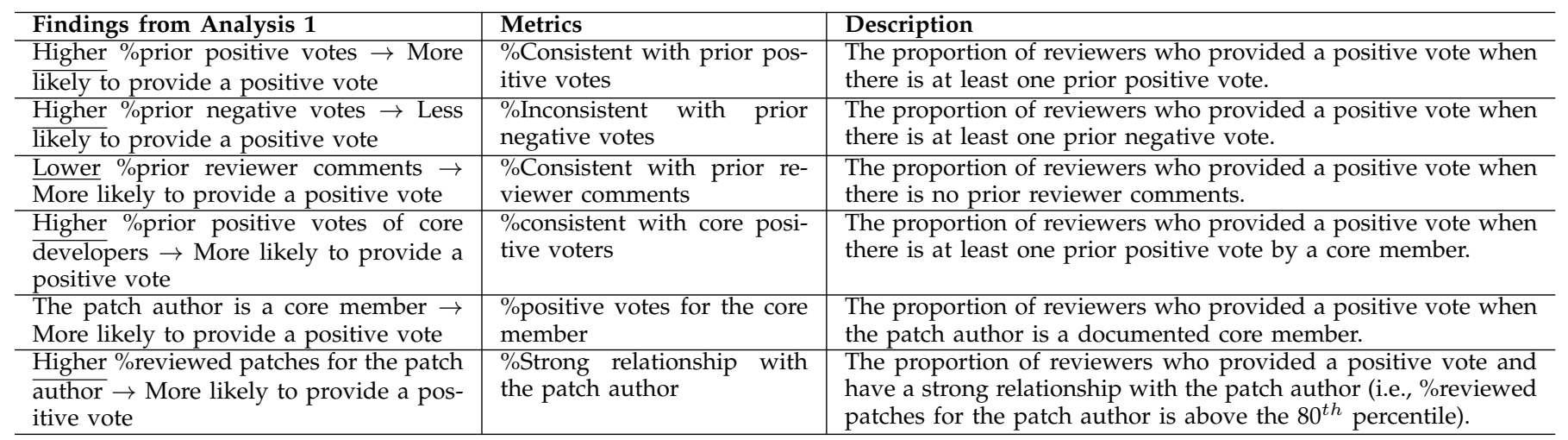

TABLE 7: Statistics of defect models. The goodness of fit for the metrics of interest $\left(\Delta \mathrm{LR} \chi^{2}\right)$ is shown in a proportion to the Overall $\mathrm{LR} \chi^{2}$.

\begin{tabular}{|c|c|c|}
\hline & OpenStack & $\mathbf{Q t}$ \\
\hline \#TRUE/FALSE Instances & $17,924 / 17,962$ & $4,857 / 14,030$ \\
\hline AUC (|Optimsm|) & $0.75(0.001)$ & $0.7(0.0002)$ \\
\hline Overall LR $\chi^{2}$ & $6,887^{* * *}$ & $1,853^{* * *}$ \\
\hline Review dynamics metrics & $225^{* * *}(3 \%)$ & $17^{*}(1 \%)$ \\
\hline \%Consistent with prior positive votes & $22^{* * *}(+)$ & $10(-)$ \\
\hline \%Inconsistent with prior negative votes & $2 \circ(+)$ & $3 \circ(-)$ \\
\hline \%Following other comments & $0 \circ(-)$ & $\dagger$ \\
\hline$\%$ Consistent with core positive voters & $40 * * *(-)$ & $4^{*}(+)$ \\
\hline$\%$ Positive votes for the core member & $158^{* * *}(-)$ & $2 \circ(+)$ \\
\hline$\%$ Strong relationship with patch author & $14^{* * *}(+)$ & $0 \circ(-)$ \\
\hline Reviewing Activities & $1,383^{* * *}(15 \%)$ & $576^{* * *}(30 \%)$ \\
\hline Patch Characteristics & $3,161^{* * *}(46 \%)$ & $1,017^{* * *}(53 \%)$ \\
\hline
\end{tabular}

\subsection{Approach}

Based on the results of Analysis 1, we define six metrics for measuring review dynamics. Table 6 describes our six metrics. We hypothesize that the higher the metric value, the more likely that a patch will be defective. To test our hypotheses, we analyze defect models that control for confounding factors. We build a simple logistic regression models to estimate the likelihood of a patch inducing fixes. Note that we only studied merged patches in Analysis 2 since we link the patches to the commits in VCS in order to identify fix-inducing patches. The review and patch characteristics described in Table 2 are used as confounding factors in our models. The response variable is assigned as TRUE if a patch induces future fixes, and FALSE otherwise. Finally, we build and analyze our defect models as described in Section 3.4

\subsection{Results}

Table 7 shows that our defect models achieve an AUC of 0.75 and 0.70 and a significant overall LR $\chi^{2}$ improvement over a null model. These results indicate that our defect models perform better than random guessing. Table 7 shows that our models yield very small AUC optimism, indicating that our models are stable enough for interpretation.

Although our review dynamics metrics are significantly associated with defect-proneness even after controlling for several confounding factors, the explanatory power of our review dynamics metrics are relatively small. Table 7 shows that our review dynamic metrics have a significant association with the likelihood of a patch inducing fixes (i.e., the $p$-value of $\Delta \mathrm{LR} \chi^{2}$ $<0.05)$. However, we observe that the review dynamics metrics did not contribute a strong explanatory power as the review and patch characteristics do. The $\Delta \mathrm{LR} \chi^{2}$ values of the review dynamics metrics account for $3 \%$ and $1 \%$ of the overall $\mathrm{LR} \chi^{2}$ of the OpenStack and $\mathrm{Qt}$ models, respectively.

We also check the proportion of patches that induced fixes for a high proportion of consistent with prior positive votes (i.e., greater $80^{\text {th }}$ percentile) for the OpenStack dataset. Then, we compare this proportion with the proportion of patches that induced fixes for a low proportion of consistent with prior positive votes (i.e., below $20^{t h}$ percentile). We find that there is little difference of the proportion of patches that induced fixes, i.e., $50 \%$ for the high metric value and $49 \%$ for the low metric value. These results suggest that despite the association between visible information about a patch under review and the evaluation decision, such associations have little impact on software quality. 
Summary: Despite the associations between visible information about a patch under review and the evaluation decision, the associations between the review dynamics metrics and the likelihood of inducing fixes are not as strong as those of confounding factors.

\section{Discussion}

We now discuss broader implications of our results with regard to our three dimensions about the visible information about a patch under review in a code review.

\subsection{Feedback}

Our results provide empirical evidence that reviewers tend to adhere to community opinions when evaluating a new proposed patch. In particular, the results of our Analysis 1 show that a reviewer is more likely to provide a positive vote if the patch has received many prior positive votes. We also find that the proportion of prior reviewer comments shares a significant relationship with the likelihood of providing a positive vote of a reviewer. These results suggest that feedback (i.e., votes and comments) of prior reviewers tends to affect an evaluation decision of a reviewer. The code review guidelines of the studied projects suggest the reviewers to focus on the technical content ${ }^{19}$ Hence, hiding the review discussion and feedback for reviewers before the reviewers provide the first feedback to the patch may allow them to focus more on the technical content of the patch. This recommended practice is also consistent with the code review practice in the traditional code inspection where reviewers are required to independently study the code changes before starting the review meeting [22].

Nevertheless, prior work shows that collaborative code reviews bring many benefits to software projects [4. 13. 42]. Studies in social science also argue that the social processes underlying the peer review process can increase the effectiveness of reviews [8, 47]. Our results of Analysis 2 also show the review dynamics have a relatively small impact on the software quality. Therefore, we believe that configuring the code review tool to be open and transparent should maximize the benefits of performing code reviews.

\subsection{Status}

While several studies report that the status of team members has an impact on code review participation and outcome [9, 59, 80], our Analysis 1 shows that the core member status of the patch author and co-reviewers share a significant association with an evaluation decision in the OpenStack dataset but not for the Qt dataset (see Table 5). One possible explanation is that reviewers may have their own strategies in reviewing. A survey by Bosu et al. reports that some reviewers prefer to evaluate patches of developers who are known to propose good

19. https://docs.openstack.org/doc-contrib-guide/ docs-review-guidelines.html patches, while some reviewers may focus on patches of newcomers [13].

\subsection{Relationship}

Our Analysis 1 shows that the proportion of reviewed patches for the patch author shares a significant association with its evaluation decision (see Table 5). Similarly, prior work finds that the historical cooperation of a reviewer with the patch author often affects the decision of whether to accept a review request [13]. These results suggest that the relationship of a reviewer with the patch author can effect the evaluation decision of the reviewer. In addition, our Analysis 2 shows that the relationship metrics are associated with the likelihood of a patch inducing fixes in OpenStack (see Table 7). Based on our findings, it is recommended that a reviewer should lessen the importance of patches from the patch authors who have a strong relationship with the reviewer. Hiding the patch author name before the reviewer provides the first feedback might allow the reviewers to focus on the technical content. Nevertheless, the hiding of the reviewer name may hinder many of the collaborative aspects of the review process [13]. Hence additional studies are needed to further understand the impact of hiding the reviewer name.

\section{ThREATS to Validity}

External Validity. We perform a case study on two open source projects. Although the OpenStack and Qt projects are commonly used as a case study of prior research [11. $13,39,43,50,74,75,83$, the results may not generalize to all software projects and all settings of the code review process. However, the goal of this paper is not to build a theory that applies to all projects, but rather to shed light that in some projects, the visible information about a patch under review can affect the evaluation decision of a reviewer. Nonetheless, additional replication studies would help to generalize our findings. To foster future replication of our study, a replication package of our work is available online. 1

Construct Validity. We extract a vote score from review messages using regular expressions in order to calculate metrics that capture visible information about a patch under review. There might be a case where the data extraction is inaccurate. However, the patterns for which we search are the activity log that is automatically generated by the Gerrit code review tool ${ }^{20}$ Moreover, we manually validate the extraction results to ensure that our regular expressions accurately identify a vote score.

We use the SZZ algorithm [64] and keyword search to identify fix-inducing patches. There might be a case where some fix-inducing patches are unidentified or the number of fixing-inducing patches is inflated by the algorithm [16, 84]. An approach that improves the

20. https://gerrit-review.googlesource.com/Documentation/ intro-gerrit-walkthrough.html 
accuracy of the SZZ algorithm [16, 82] may further improve the accuracy of our results.

When we examine the relationship between review dynamics and software quality, we focus on the review dynamics in merged reviews. The discovered relationships may be impacted when considering review dynamics in abandoned reviews. However, to determine whether a review induced fixes in the historical data, we need to link the review to the associated commit in VCS. Since the abandoned reviews in the code review tool are not integrated into the VCS, we cannot determine whether the abandoned reviews induced fixes or not. Hence, we can only use merged reviews when we investigate whether the review dynamics have an impact on software quality.

Internal Validity. Our results are derived from the statistical models that we fitted to our data. However, the relationship in our statistical models does not represent the causal effects of the review dynamics on the evaluation decision and on software quality. Hence, qualitative or experimental studies are needed in order to better understand the reasons and effects of review dynamics.

There might be other factors that influence the evaluation decision of a reviewer. For example, prior survey studies report that the quality of a proposed patch has an impact on the evaluation decision of the patch [13, 35]. Yet, the quality of a patch can be defined in many aspects, e.g., code correctness, readability, and maintainability [35]. In our study, we address this concern by using patch characteristics that are commonly known to share a link to the evaluation decision of the patch and software quality as confounding factors in our models [7, 26, 32, 43, 73].

Nonetheless, it might still be possible that patches are good at the first submission. Then, many reviewers provided positive votes which can be considered as providing a vote consistent with the prior positive voters. To address this concern, we use one-sided MannWhitney $U$ tests to examine whether the proportion of reviewers who provided a consistent vote with prior positive voters (i.e., \%positive votes) of the patches with one revision is statistically greater than that of the patches with multiple revisions. We find that \%positive votes of the patches with one revision is not significantly greater than that of the patches with multiple revisions.

An overfit model may exaggerate spurious relationships between explanatory and response variables. Babyak pointed out that automated variable selection (e.g., forward stepwise selection), pretesting of candidate predictors by checking the univariate relation between each variable and the response, and dichotomization of continuous variables can pose a considerable risk for spurious findings in models [3]. To mitigate this concern, we follow the modeling approach of Harrell Jr. to carefully avoid the likelihood of overfitting in our models [29].

\section{Conclusions}

Code reviews processes nowadays are often performed in a transparent environment, where various information (e.g., review comments) are visible, in order to enhance active and timely collaboration. However, such visible information may have an impact on code review practices and their effectiveness. Hence, in this paper, we first investigate the review dynamics, i.e., a practice of providing an evaluation decision by a reviewer. To do so, we define eight metrics that capture the visible information about a patch under review and analyze their associations with the likelihood that a reviewer will provide a positive vote to accept the patch. Then, we further investigate whether the uncovered review dynamics are associated with defect-proneness. Through a case study of the OpenStack and Qt projects, we find that:

- The proportion of prior positive votes and prior reviewer comments are highly associated with the evaluation decision of a reviewer.

- The co-working frequency of the reviewer with the patch author also have a positive association with the likelihood of providing a vote to accept a patch.

- While we have controlled for several confounding factors, the review dynamics metrics, e.g., the proportion of reviewers who provided a positive vote and the proportion of reviewers who have a strong relationship with the patch author, have a relatively small association with defect-proneness.

The key contribution of this work is to highlight that the visible information in the code review tools implicitly influences the evaluation decision of a reviewer. Our findings are derived from two large open source projects (OpenStack and Qt) which actively use tool-based code review processes. However, the goal of this paper is not to define a wide ranging theory that holds for every setting. We expect reviewing practices to vary from project to project based on the social and technical norms of the team. Nevertheless, we believe that our findings would be still of value to teams in managing and improving their code review processes.

\section{REFERENCES}

[1] G. S. Aikenhead, "Collective decision making in the social context of science," Science Education, vol. 69, no. 4, pp. 453-475, 1985.

[2] A. Aurum, H. Petersson, and C. Wohlin, "State-of-the-Art: Software Inspections After 25 Years," Software Testing, Verification and Reliability, vol. 12, no. 3, pp. 133-154, 2002.

[3] M. A. Babyak, "What You See May Not Be What You Get: A Brief, Nontechnical Introduction to Overfitting in Regression-Type Models," Psychosomatic Medicine, vol. 66, no. 3, pp. 411-421, 2004.

[4] A. Bacchelli and C. Bird, "Expectations, Outcomes, and Challenges Of Modern Code Review," in ICSE, 2013, pp. 712-721.

[5] G. Bavota and B. Russo, "Four Eyes Are Better Than Two: On the Impact of Code Reviews on Software Quality," in ICSME, 2015, pp. 81-90.

[6] O. Baysal, O. Kononenko, R. Holmes, and M. W. Godfrey, "The Secret Life of Patches: A Firefox Case Study," in WCRE, 2012, pp. 447-455. 
[7] _ " "Investigating Technical and Non-Technical Factors Influencing Modern Code Review," EMSE, vol. 21, no. 3, pp. 932-959, 2015.

[8] A. G. Bedeian, "Peer review and the social construction of knowledge in the management discipline." Academy of Management Learning E Education, vol. 3, no. 2, pp. 198 - 216, 2004.

[9] C. Bird, A. Gourley, P. Devanbu, M. Gertz, and A. Swaminathan, "Mining Email Social Networks," in MSR, 2006, pp. 137-143.

[10] C. Bird, N. Nagappan, B. Murphy, H. Gall, and P. Devanbu, "Don't Touch My Code! Examining the Effects of Ownership on Software Quality," in ESEC/FSE, 2011, pp. 4-14.

[11] A. Bosu and J. Carver, "Impact of Developer Reputation on Code Review Outcomes in OSS Projects: An Empirical Investigation," in ESEM, 2014, pp. 33-42.

[12] A. Bosu, J. Carver, R. Guadagno, B. Bassett, D. McCallum, and L. Hochstein, "Peer Impressions in Open Source Organizations: A survey," JSS, vol. 94, pp. 4-15, 2014.

[13] A. Bosu, J. C. Carver, C. Bird, J. Orbeck, and C. Chockley, "Process Aspects and Social Dynamics of Contemporary Code Review: Insights from Open Source Development and Industrial Practice at Microsoft," TSE, vol. 43, no. 1, pp. 56-75, 2017.

[14] J. S. Bunderson and R. E. Reagans, "Power, status, and learning in organizations," Organization Science, vol. 22, no. 5, pp. 1182-1194, 2011.

[15] J. C. Carver, B. Caglayan, M. Habayeb, B. Penzenstadler, and A. Yamashita, "Collaborations and Code Reviews," IEEE Software, vol. 32, no. 5, pp. 27-29, 2015.

[16] D. A. Da Costa, S. McIntosh, W. Shang, U. Kulesza, R. Coelho, and A. E. Hassan, "A Framework for Evaluating the Results of the SZZ Approach for Identifying Bug-Introducing Changes," TSE, vol. 43, no. 7, pp. 641-657, 2017.

[17] L. Dabbish, C. Stuart, J. Tsay, and J. Herbsleb, "Social Coding in GitHub: Transparency and Collaboration in an Open Software Repository," in CSCW, 2012, pp. 1277-1286.

[18] M. D'Ambros, M. Lanza, and R. Robbes, "Evaluating defect prediction approaches: A benchmark and an extensive comparison," EMSE, vol. 17, no. 4-5, pp. 531-577, 2012.

[19] A. O. De Guinea, J. Webster, and D. S. Staples, "A meta-analysis of the consequences of virtualness on team functioning," Information E Management, vol. 49, no. 6, pp. 301-308, 2012.

[20] M. Deutsch and H. B. Gerard, "A study of normative and informational social influences upon individual judgment." The journal of abnormal and social psychology, vol. 51, no. 3, p. 629, 1955.

[21] B. Efron, "How Biased is the Apparent Error Rate of a Prediction Rule?" Journal of the American Statistical Association, vol. 81, no. 394, pp. 461-470, 1986.

[22] M. E. Fagan, "Design and Code Inspections to Reduce Errors in Program Development," IBM System Journal, vol. 15, no. 3, pp. 182-211, sep 1976.

[23] S. Fuller, Knowledge management foundations. Routledge, 2012.

[24] D. M. German, G. Robles, G. Poo-Caamaño, X. Yang, H. Iida, and K. Inoue, "'Was my contribution fairly reviewed ?" A Framework to Study the Perception of Fairness in Modern Code Reviews," in ICSE, no. 2, 2018, pp. 523-534.

[25] C. L. Goues, Y. Brun, S. Apel, E. Berger, S. Khurshid, and Y. Smaragdakis, "Effectiveness of Anonymization in Double-Blind Review," Communications of the ACM, vol. 61, no. 6, pp. 30-33, 2018.

[26] G. Gousios, M. Pinzger, and A. van Deursen, "An Exploratory Study of the Pull-based Software Development Model," in ICSE, 2014, pp. 345-355.

[27] I. Hames, Peer Review and Manuscript Management in Scientific Journals: Guidelines for Good Practice. John Wiley \& Sons, 2007.

[28] J. A. Hanley and B. J. McNeil, "The Meaning and Use of the Area under a Receiver Operating Characteristic (ROC) Curve," Radiology, vol. 143, no. 1, pp. 29-36, 1982.

[29] F. E. Harrell Jr., Regression Modeling Strategies: With Application to Linear Models, Logistic Regression, and Survival Analysis, 2nd ed. Springer, 2015.

[30] A. E. Hassan, "Predicting Faults Using the Complexity of Code
Changes," in ICSE, 2009, pp. 78-88.

[31] J. P. Huelsenbeck and K. A. Crandall, "Phylogeny Estimation and Hypothesis Testing Using Maximum Likelihood," Annu. Rev. Ecol. Evol. Syst., vol. 28, pp. 437-466, 1997.

[32] Y. Jiang, B. Adams, and D. M. German, "Will My Patch Make It? And How Fast? Case Study on the Linux Kernel," in MSR, 2013, pp. 101-110.

[33] J. Jiarpakdee, C. Tantithamthavorn, and C. Treude, "Autospearman: Automatically mitigating correlated metrics for interpreting defect models," in ICSME, 2018, pp. 92-103.

[34] Y. Kamei, E. Shihab, B. Adams, A. E. Hassan, A. Mockus, A. Sinha, and N. Ubayashi, "A Large-Scale Empirical Study of Just-in-Time Quality Assurance," TSE, vol. 39, no. 6, pp. 757-773, 2013.

[35] O. Kononenko, O. Baysal, and M. W. Godfrey, "Code Review Quality: How Developers See It," in ICSE, 2016, pp. 1028-1038.

[36] O. Kononenko, O. Baysal, L. Guerrouj, Y. Cao, and M. W. Godfrey, "Investigating Code Review Quality: Do People and Participation Matter?" in ICSME, 2015, pp. 111-120.

[37] M. Lamont and J. Guetzkow, How Quality Is Recognized by Peer Review Panels: The Case of the Humanities. Cham: Springer International Publishing, 2016, pp. 31-41.

[38] R. P. Larrick, "The social context of decisions," Annual review of organizational psychology and organizational behavior, vol. 3, pp. 441467, 2016.

[39] A. Lee, J. C. Carver, and A. Bosu, "Understanding the Impressions, Motivations, and Barriers of One Time Code Contributors to FLOSS Projects: A Survey," in ICSE, 2017, pp. 187-197.

[40] S. D. McClurg, "The Electoral Relevance of Political Talk: Examining Disagreement and Expertise Effects in Social Networks on Political Participation," AJPS, vol. 50, no. 3, pp. 737-754, 2006.

[41] J. McConnell, "Mozilla experiment aims to reduce bias in code reviews," 2018. [Online]. Available: https://blog.mozilla.org/ blog/2018/03/08/gender-bias-code-reviews/

[42] N. McDonald and S. Goggins, "Performance and Participation in Open Source Software on GitHub," in CHI - Extended Abstract, 2013, pp. 139-144.

[43] S. McIntosh and Y. Kamei, "Are Fix-Inducing Changes a Moving Target ? A Longitudinal Case Study of Just-In-Time Defect Prediction," TSE, vol. 44, no. 5, pp. 412 - 428, 2017.

[44] S. McIntosh, Y. Kamei, B. Adams, and A. E. Hassan, “The Impact of Code Review Coverage and Code Review Participation on Software Quality," in Proceedings of MSR, 2014, pp. 192-201.

[45] _ " "An Empirical Study of the Impact of Modern Code Review Practices on Software Quality," EMSE, vol. 21, no. 5, pp. 21462189, 2016.

[46] J. R. Mesmer-Magnus, L. A. DeChurch, M. Jimenez-Rodriguez, J. Wildman, and M. Shuffler, "A meta-analytic investigation of virtuality and information sharing in teams," Organizational Behavior and Human Decision Processes, vol. 115, no. 2, pp. 214-225, 2011.

[47] J. A. Minson, J. S. Mueller, and R. P. Larrick, "The contingent wisdom of dyads: When discussion enhances vs. undermines the accuracy of collaborative judgments," Management Science, vol. 64, no. 9, pp. 4177-4192, 2017.

[48] R. Morales, S. McIntosh, and F. Khomh, "Do Code Review Practices Impact Design Quality? A Case Study of the Qt, VTK, and ITK Projects," in SANER, 2015, pp. 171-180.

[49] Á. Münnich, G. Maksa, and R. J. Mokken, "Collective judgement: combining individual value judgements," Mathematical Social Sciences, vol. 37, no. 3, pp. 211-233, 1999.

[50] T. Pangsakulyanont, P. Thongtanunam, D. Port, and H. Iida, "Assessing MCR Discussion Usefulness using Semantic Similarity," in IWESEP, 2014, pp. 49-54.

[51] C. Pavitt, "An interactive input-process-output model of social influence in decision-making groups," Small Group Research, vol. 45, no. 6, pp. 704-730, 2014.

[52] J. Pissarra and J. C. Jesuino, "Idea generation through computermediated communication: The effects of anonymity," Journal of Managerial Psychology, vol. 20, no. 3/4, pp. 275-291, 2005.

[53] A. L. Porter and F. A. Rossini, "Peer review of interdisciplinary 
research proposals," Science, Technology, and Human Values, vol. 10, no. 3, pp. 33-38, 1985.

[54] P. Radostina K., "Face-to-face versus virtual teams: What have we really learned?." The Psychologist-Manager Journal, no. 1, p. 2, 2014.

[55] F. Rahman and P. Devanbu, "How, and why, process metrics are better," in ICSE, 2013, pp. 432-441.

[56] P. C. Rigby and C. Bird, "Convergent Contemporary Software Peer Review Practices," in ESEC/FSE, 2013, pp. 202-212.

[57] P. C. Rigby, D. M. German, L. Cowen, and M.-a. Storey, “Peer Review on Open-Source Software Projects: Parameters, Statistical Models, and Theory," TOSEM, vol. 23, no. 4, pp. 35:1-35:33, 2014.

[58] P. C. Rigby, D. M. German, and M.-A. Storey, “Open Source Software Peer Review Practices: A Case Study of the Apache Server," in ICSE, 2008, pp. 541-550.

[59] P. C. Rigby and M.-A. Storey, "Understanding Broadcast Based Peer Review on Open Source Software Projects," in ICSE, 2011, pp. 541-550.

[60] C. Sadowski, E. Söderberg, L. Church, M. Sipko, and A. Bacchelli, "Modern Code Review: A Case Study at Google," in ICSECompanion, 2018, pp. 181-190.

[61] W. Scacchi, "Free/Open Source Software Development: Recent Research Results and Methods," Adv. Electr. Comput. Eng., vol. 69, no. 6, pp. 243-295, 2007.

[62] M. Seeber and A. Bacchelli, "Does single blind peer review hinder newcomers?." SCIENTOMETRICS, vol. 113, no. 1, pp. 567 - 585, 2017.

[63] J. Shimagaki, Y. Kamei, S. McIntosh, A. E. Hassan, and N. Ubayashi, "A Study of the Quality-Impacting Practices of Modern Code Review at Sony Mobile," in ICSE - Companion, 2016, pp. 212-221.

[64] J. Śliwerski, T. Zimmermann, and A. Zeller, "When do changes induce fixes?" in MSR, 2005, pp. 1-5.

[65] D. Spadini, M. Aniche, and A. Bacchelli, "PyDriller: Python framework for mining software repositories," in ESEC/FSE, 2018, pp. 908-911.

[66] G. Stasser and W. Titus, "Hidden profiles: A brief history," Psychological Inquiry, vol. 14, no. 3-4, pp. 304-313, 2003.

[67] M. R. Steenbergen and S. J. Bradford, "Modeling Multilevel Data Structures," AJPS, vol. 46, no. 1, pp. 218-237, 2002.

[68] I. Steinmacher, T. U. Conte, M. A. Gerosa, and D. F. Redmiles, "Social Barriers Faced by Newcomers Placing Their First Contribution in Open Source Software Projects," in CSCW, 2015, pp. 1379-1392.

[69] E. Suhay, "Explaining Group Influence: The Role of Identity and Emotion in Political Conformity and Polarization," Political Behav., vol. 37 , no. 1 , pp. $221-251,2015$

[70] C. Tantithamthavorn and A. E. Hassan, "An experience report on defect modelling in practice," in ICSE-Companion, 2018, pp. 286295.

[71] C. Tantithamthavorn, S. McIntosh, A. E. Hassan, and K. Matsumoto, "An empirical comparison of model validation techniques for defect prediction models," TSE, vol. 43, no. 1, pp. 1-18, 2017.

[72] P. Thongtanunam, S. McIntosh, A. E. Hassan, and H. Iida, "Investigating Code Review Practices in Defective Files: An Empirical Study of the Qt System," in MSR, 2015, pp. 168-179.

[73] —, "Revisiting Code Ownership and its Relationship with Software Quality in the Scope of Modern Code Review," in ICSE, 2016, pp. 1039-1050.

[74] — " "Review Participation in Modern Code Review," EMSE, [77] A. Tomkins, M. Zhang, and W. D. Heavlin, "Reviewer bias in vol. 22, no. 2, pp. 768-817, 2017.

[75] P. Thongtanunam, C. Tantithamthavorn, R. G. Kula, N. Yoshida, H. Iida, and K.-i. Matsumoto, "Who Should Review My Code? A File Location-Based Code-Reviewer Recommendation Approach for Modern Code Review," in SANER, 2015, pp. 141-150.

[76] P. Thongtanunam, X. Yang, N. Yoshida, R. G. Kula, C. C. Ana Erika, K. Fujiwara, and H. Iida, "ReDA: A Web-based Visualization Tool for Analyzing Modern Code Review Dataset," in ICSME, 2014, pp. 606-609.

single- versus double-blind peer review," NAS, vol. 114, no. 48, pp. 12 708-12713, 2017.

[78] G. D. L. Travis and H. M. Collins, "New light on old boys: Cognitive and institutional particularism in the peer review system," Science, Technology, and Human Values, vol. 16, no. 3, pp. 322-341, 1991.

[79] J. Tsay, L. Dabbish, and J. Herbsleb, "Influence of Social and Technical Factors for Evaluating Contribution in GitHub," in ICSE, 2014, pp. 356-366.

[80] —, "Let's Talk About It: Evaluating Contributions through Discussion in GitHub," in FSE, 2014, pp. 144-154.

[81] B. Vasilescu, Y. Yu, H. Wang, P. Devanbu, and V. Filkov, “Quality and Productivity Outcomes Relating to Continuous Integration in GitHub," in ESEC/FSE, 2015, pp. 805-816.

[82] R. Wu, H. Zhang, S. Kim, and S. Cheung, "ReLink: Recovering Links between Bugs and Changes," in FSE/ECSE, 2011, pp. 15-25.

[83] X. Xia, D. Lo, X. Wang, and X. Yang, "Who Should Review This Change? Putting Text and File Location Analyses Together for More Accurate Recommendations," in ICSME, 2015, pp. 261-270.

[84] S. Yatish, J. Jiarpakdee, P. Thongtanunam, and C. Tantithamthavorn, "Mining Software Defects: Should We Consider Affected Releases?" in ICSE, 2019, pp. 654-665.

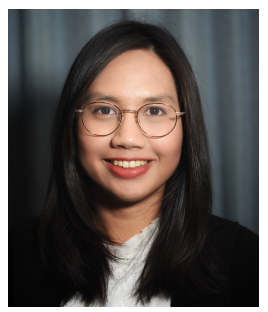

Patanamon Thongtanunam is a lecturer at the School of Computing and Information Systems, the University of Melbourne, Australia. Prior to that, she was a research fellow of Japan Society for the Promotion of Science (JSPS). She received $\mathrm{PhD}$ in Information Science from Nara Institute of Science and Technology, Japan. Her research interests include empirical software engineering, mining software repositories, software quality, and human aspect. Her research has been published at top-tier software engineering venues like International Conference on Software Engineering (ICSE) and Journal of Empirical Software Engineering (EMSE). More about Patanamon and her work is available online at http://patanamon.com

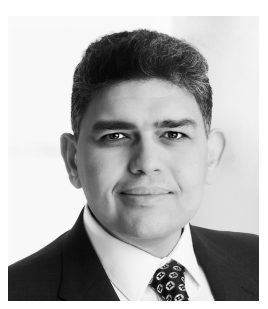

Ahmed E. Hassan is an IEEE Fellow, an ACM SIGSOFT Influential Educator, an NSERC Steacie Fellow, the Canada Research Chair (CRC) in Software Analytics, and the NSERC/BlackBerry Software Engineering Chair at the School of Computing at Queen's University, Canada. His research interests include mining software repositories, empirical software engineering, load testing, and log mining. He received a $\mathrm{PhD}$ in Computer Science from the University of Waterloo. He spearheaded the creation of the Mining Software Repositories (MSR) conference and its research community. He also serves/d on the editorial boards of IEEE Transactions on Software Engineering, Springer Journal of Empirical Software Engineering, and PeerJ Computer Science. Contact: ahmed@cs.queensu.ca. More information at: http://sail.cs.queensu.ca/ 


\section{University Library}

\section{- M M N E R VA A gateway to Melbourne's research publications}

Minerva Access is the Institutional Repository of The University of Melbourne

Author/s:

Thongtanunam, P;Hassan, AE

Title:

Review Dynamics and Their Impact on Software Quality

Date:

2020

Citation:

Thongtanunam, P. \& Hassan, A. E. (2020). Review Dynamics and Their Impact on Software Quality. IEEE Transactions on Software Engineering, 47 (12), pp.1-1. https://doi.org/10.1109/ tse.2020.2964660.

Persistent Link:

http://hdl.handle.net/11343/267357 